University of Wollongong

Research Online

Faculty of Informatics - Papers (Archive)

Faculty of Engineering and Information

Sciences

27-8-2007

\title{
A Study of Different Angles for Higher Order Rotation Spreading Matrix for BSOFDM in UWB Channels
}

\author{
Ibrahim S. Raad \\ University of Wollongong, ibrahim@uow.edu.au \\ Xiaojing Huang \\ University of Wollongong, huang@uow.edu.au \\ R. Raad \\ University of Wollongong, raad@uow.edu.au
}

Follow this and additional works at: https://ro.uow.edu.au/infopapers

Part of the Physical Sciences and Mathematics Commons

\section{Recommended Citation}

Raad, Ibrahim S.; Huang, Xiaojing; and Raad, R.: A Study of Different Angles for Higher Order Rotation Spreading Matrix for BSOFDM in UWB Channels 2007.

https://ro.uow.edu.au/infopapers/637

Research Online is the open access institutional repository for the University of Wollongong. For further information contact the UOW Library: research-pubs@uow.edu.au 


\title{
A Study of Different Angles for Higher Order Rotation Spreading Matrix for BSOFDM in UWB Channels
}

\author{
Abstract \\ This paper presents a study into different angles for higher order Rotation spreading matrix developed for \\ BSOFDM. It was shown previously that for block size $M=2$ that the angle $\alpha=\pi / 3$ achieved the best result \\ in terms of BER in UWB channels. It was discovered that this was no longer the case when the higher \\ order Rotation spreading matrix was used for larger $\mathrm{M}$ sized blocks and that other angles produced better \\ results which proves that the Rotation spreading matrix advantage over existing spreading matrices such \\ as the Hadamard is its flexibility to be adapted to different communication systems.

\section{Keywords} \\ OFDM, Rotation Spreading Matrix, Block Spread-OFDM, UWB

\section{Disciplines} \\ Physical Sciences and Mathematics

\section{Publication Details} \\ This conference paper was originally published as Raad, IS, Huang, X, Raad, R, A Study of Different Angles \\ for Higher Order Rotation Spreading Matrix for BSOFDM in UWB Channels, 2nd International Wireless \\ Broadband and Ultra Wideband Communications AusWireless 2007, 27-30 Aug, 18-18.
}




\title{
A Study of Different Angles for Higher Order Rotation Spreading Matrix for BSOFDM in UWB Channels
}

\author{
Ibrahim S. Raad, Xiaojing Huang and Raad Raad \\ School of Electrical, Computer and Telecommunications Engineering \\ University of Wollongong, N.S.W Australia \\ ibrahim@uow.edu.au
}

\begin{abstract}
This paper presents a study into different angles for higher order Rotation spreading matrix developed for BSOFDM. It was shown previously that for block size $M=2$ that the angle $\alpha=\frac{\pi}{3}$ achieved the best result in terms of BER in UWB channels. It was discovered that this was no longer the case when the higher order Rotation spreading matrix was used for larger $M$ sized blocks and that other angles produced better results which proves that the Rotation spreading matrix advantage over existing spreading matrices such as the Hadamard is its flexibility to be adapted to different communication systems. ${ }^{1}$
\end{abstract}

Key Words-OFDM, Rotation Spreading Matrix, Block Spread-OFDM, UWB

\section{Introduction}

Many solutions have been presented to allow a communications system to improve its spectral efficiency of the modulation schemes by applying different schemes such as adaptive modulation based on the Bit Error Rate (BER) or the signal to noise ratio. But these systems suffer from complexity issues and the fact many still, after varies algorithms to improve the spectral efficiency, end up using BPSK or QPSK. In [1] a new matrix called the Rotation spreading matrix was introduced and in frequency selective channels such as the UWB channels it was shown to outperform other spreading matrices like the Hadamard which can be used in Block Spread OFDM (BSOFDM) and is discussed in detail in [2], [3], [4], [5] and [6]. In [7] it was experimentally shown for a block size of $M=2$ the angle $\alpha=\frac{\pi}{3}$ was shown in terms of BER to outperform other angles. But this is no longer the case for higher order Rotation spreading matrix discussed in [8]. As will be shown below for larger block sizes of $M$, there are more advantages in using other angles.

This paper is organized as follows. Section 2 provides a description of the system used to test the higher order Rotation spreading matrix for BSOFDM. Section 3 discusses

\footnotetext{
${ }^{1}$ This research is sponsored by ARC DP 0558405
}

the Rotation spreading matrix used in this study and discusses the new constellation points achievable for block size of $M=4$. A brief description is given of the four UWB channel IEEE defined models in Section 4. Section 5 gives the results achieved with the Rotation spreading matrix and the different angles over the UWB channels. And finally in Section 6 a conclusion is given and future recommendations for this work.

\section{System Description}

The Rotation spreading matrix is used in BSOFDM to spread the data across the $M$ sized blocks to ensure it achieves frequency diversity in frequency selective channels such as the UWB channels [2], [3], [4] and [5]. The BSOFDM channel model is shown in Figure 1.

$$
y=C q+n
$$

The output of the receiver's FFT processor is shown in Equation 1, where $y$ is the FFT output, $q \in A^{N}$ is the vector of transmitted symbols, each drawn from an alphabet $A, C$ is a diagonal matrix of complex normal fading coefficients, and $n$ is a zero mean complex normal random vector. Equalization of the received data is done through multiplication by $C^{-1}$ and then "quantized independently on each subcarrier to form the soft or hard decision $\hat{q}$ which may be further processed if the data bits are coded" [4]. There is no loss in performance when the detection is performed independently on each carrier due to the noise being independent and identically distributed with fading been diagonal [4].

The block spreading matrices are used to introduce dependence among the subcarriers. $N$ subcarriers are split into $\frac{N}{M}$ of blocks of size $M$, where $M$ size blocks can be any size and the Rotation spreading matrix is $M \times M$. The length $M$ output vectors are interleaved using general block interleaving to ensure the symbols are statistically independent so as to encounter independent fading channels. This will ensure in a dispersive frequency selective channel the data is statistically less likely to become corrupted and studies and simulations have shown this to be correct. 


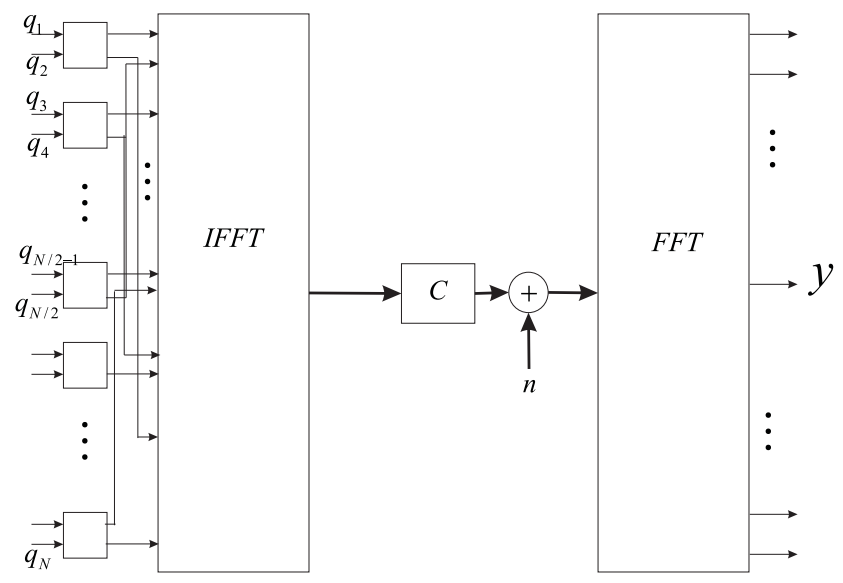

Figure 1. Block diagram representation of the BSOFDM channel for a block length of two [4].

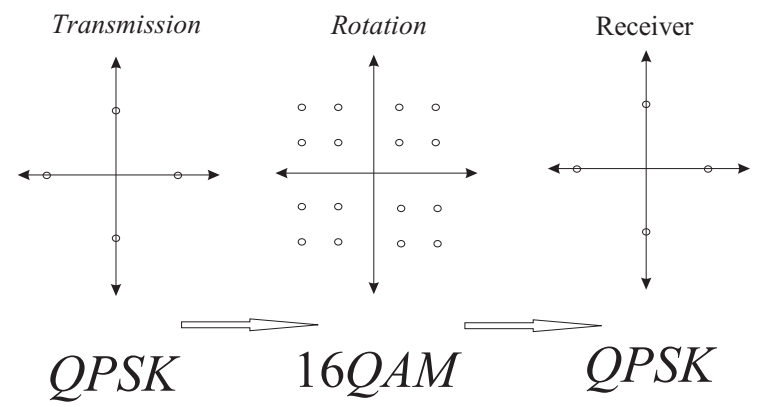

Figure 2. The process through which the transmitted modulation is converted into a higher order modulation and then returned at the receiver.

The transmitter's IFFT has the interleaved data passed through it and this data is sent across the frequency selective channel. The data is passed through an FFT processor at the receiver and deinterleaved before using block by block processing.

The spreading matrices are generally used to increase the correlation between the transmitted symbols after the transmission has occurred. Unlike adaptive modulation schemes where depending on the system, a higher order modulation scheme is used to retransmit the data depending on the conditions presented, BSOFDM utilizes spreading matrices to increase the correlation between the transmitted symbols, rather than retransmitting. This is depicted in Figure 2. This paper continues the study of the Rotation spreading matrix presented in [1] and [7] and studies different angles for higher order block sizes. Below the description of the Rotation spreading matrix is presented and discussed in terms of the different possible combinations achievable for higher order block sizes such as $M=4$.

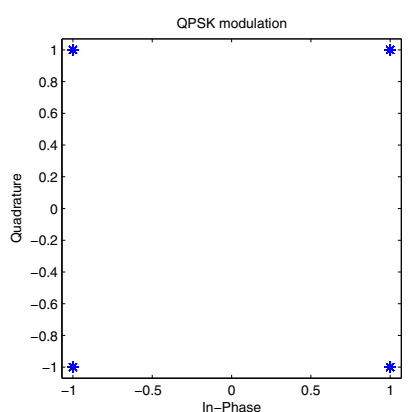

Figure 3. The QPSK constellation points.

\section{Rotation spreading matrix and Varying angles for $M=4$}

The Rotation spreading matrix BSOFDM was proposed in [1] and its structure is as follows for $U_{2}=2 \times 2$,

$$
U=\left[\begin{array}{cc}
1 & \tan (\alpha) \\
\tan (\alpha) & -1
\end{array}\right]
$$

In [8] the higher order structure was presented and has the following structure for $U_{4}=4 \times 4$,

$U_{4}=\left[\begin{array}{cccc}1 & \tan (\alpha) & 1 & \tan (\alpha) \\ \tan (\alpha) & -1 & \tan (\alpha) & -1 \\ 1 & \tan (\alpha) & -1 & -\tan (\alpha) \\ \tan (\alpha) & -1 & -\tan (\alpha) & 1\end{array}\right]$

With this Rotation spreading matrix for block spread OFDM, the following figures depict the varying constellation points which are achievable for higher block size $M=4$. Figure 3 depicts the constellation scatter plot of QPSK which will be used for this study. Figures 4, 5, 6,7 and 8 depict the different constellation points which are achievable using the angles $\alpha=\frac{\pi}{3}, \frac{\pi}{6}, \frac{\pi}{5}, \frac{\pi}{7}$ and $\frac{\pi}{9}$ for block size $M=4$ with the number of subcarriers of $N=512$.

An interesting feature of this higher order Rotation spreading matrix is for angles such as $\alpha=\frac{\pi}{4}$, the modulation constellation no longer rotates on it self as it did with the case of block size $M=2$ in [7]. As can be seen from Figure 10, it still increased the correlation between the transmitted symbols. This was also true for the angles $\frac{p i}{2}$ and $\pi$ which for $M=2$ did nothing to improve the correlation of the symbols transmitted. This can be seen in Figures 11 and 12.

\section{UWB channels}

Ultra-wideband (UWB) systems occupy - by definition - a signal spectrum of more than $500 \mathrm{MHz}$ or more than $20 \%$ with regards to their centre frequency. The application of such large bandwidths enables communication 


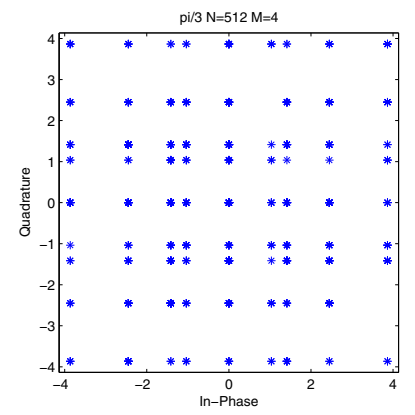

Figure 4. Higher Order Rotation spreading matrix with rotation $\alpha=\frac{\pi}{3} M=4 N=512$.

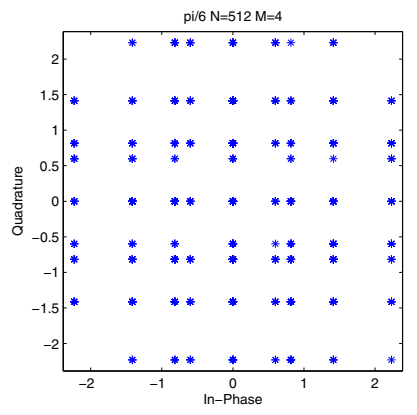

Figure 5. Higher Order Rotation spreading matrix with rotation $\alpha=\frac{\pi}{6} M=4 N=512$.

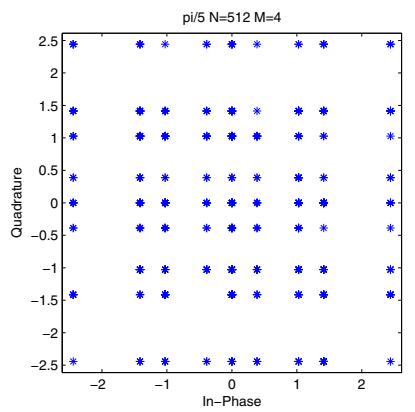

Figure 6. Higher Order Rotation spreading matrix with rotation $\alpha=\frac{\pi}{5} M=4 N=512$.

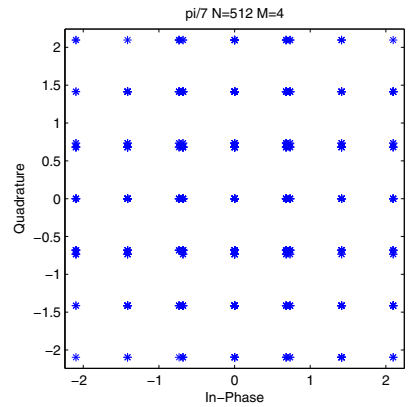

Figure 7. Higher Order Rotation spreading matrix with rotation $\alpha=\frac{\pi}{7} M=4 N=512$.

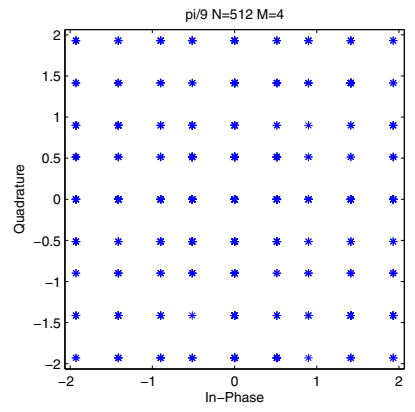

Figure 8. Higher Order Rotation spreading matrix with rotation $\alpha=\frac{\pi}{9} M=4 N=512$.

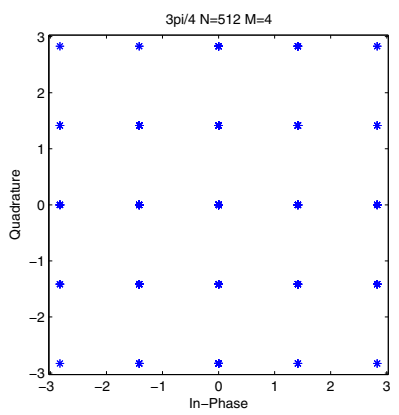

Figure 9. Higher Order Rotation spreading matrix with rotation $\alpha=\frac{3 \pi}{4} M=4 N=512$. 


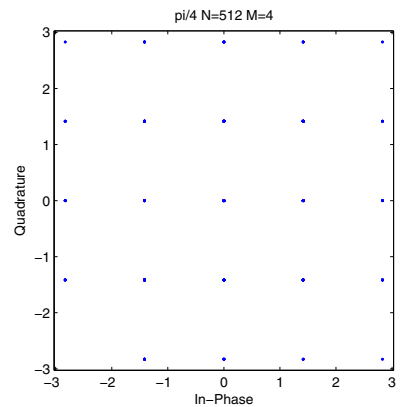

Figure 10. Higher Order Rotation spreading matrix with rotation $\alpha=\frac{\pi}{4} M=4 N=512$.

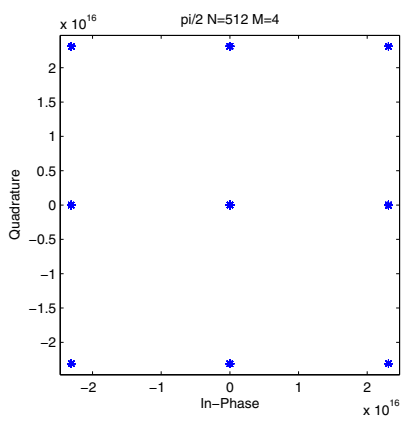

Figure 11. Higher Order Rotation spreading matrix with rotation $\alpha=\frac{\pi}{2} M=4 N=512$.

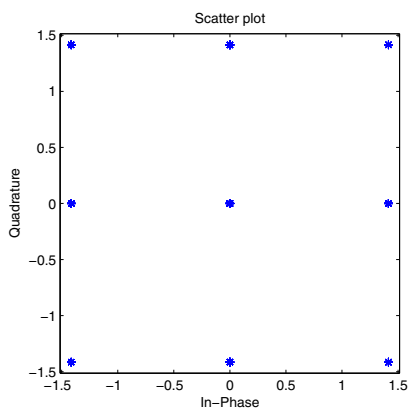

Figure 12. Higher Order Rotation spreading matrix with rotation $\alpha=\pi M=4 N=512$.

\begin{tabular}{|c|c|c|c|c|}
\hline & CM1 & CM2 & CM3 & CM4 \\
\hline Channel Model & LOS & NLOS & NLOS & NLOS \\
& $0-4 m$ & $0-4 m$ & $4-10 m$ & $10 m$ \\
Mean Ex.delay(ns) & 5 & 9.9 & 15.9 & 30.1 \\
RMS Delay(ns) & 5 & 8.0 & 15.0 & 25.0 \\
No.of multi-arrival & 12.5 & 15.3 & 24.9 & 41.2 \\
within 10dB of peak & & & & \\
\hline
\end{tabular}

Table 1. UWB channels defined by IEEE [9]

systems with unique novel properties, like high-precision indoor positioning.

Based on the Saleh-Valenzuela model for indoor multipath radio propagation channels, a set of statistically UWB channel models has been produced by the IEE E.802.15.3a task group [9]. Line of Sight (LOS) and Non-Line of Sight (NLOS) cases can be modelled using these standard channel models. The four different scenarios are summarized in table 1. Each are identified from $C M 1$ to $C M 4$. These channels are used in our study of the various angles for higher order Rotation spreading matrix.

\section{Results}

It can be shown that for higher Order Rotation spreading matrix that different angles outperform in terms of BER the angle shown in [7] to have the best performance for a two by two matrix. It can also be shown that where previously angles such as $\alpha=\frac{\pi}{4}, \alpha=\frac{\pi}{2}$ and $\alpha=\pi$ could not be used for a block size of $M=2$, since this just rotated the modulation back onto it self, for higher order Rotation spreading matrix however it showed they increased the correlation between the transmitted symbols and the angle $\alpha=\frac{\pi}{4}$ outperformed the other angles such as $\frac{\pi}{3}$ in UWB channels and can be seen in Figure 13. It was shown that this angle does not improve for block size $M=2$ in [7]. While the two angles $\alpha=\frac{p i}{2}, \pi$ showed improvement over other spreading matrices they achieved the worst results from the angles studied and can be seen in Figure 14.

For angle $\alpha=\frac{3 \pi}{4}$ the Rotation spreading matrix for higher order in $C M 3$ achieved the best results and can be seen below in Figure 15. Also angle $\alpha=\frac{\pi}{5}$ produced good results and can be seen in Figure 16 in UWB channel $C M 3$ and in Figure 17 in UWB channel $C M 1$.

So the advantage of this Rotation spreading matrix in higher order block sizes is its flexibility in adapting to the required communication environment. When the block size $M=2$ was used in [7] it was shown that some angles do not improve the performance of the system but for higher order matrix the very same angles showed that they can be used and a better BER is achievable. The channels used for this study is UWB $C M 1$ to $C M 4$ described in Table 1. The subcarriers used varied from $N=64$ to $N=512$. The block size was $M=4$ and the decoder at the receiver used was Zero Forcing assuming that the channel was known. Not all results are shown due to space 


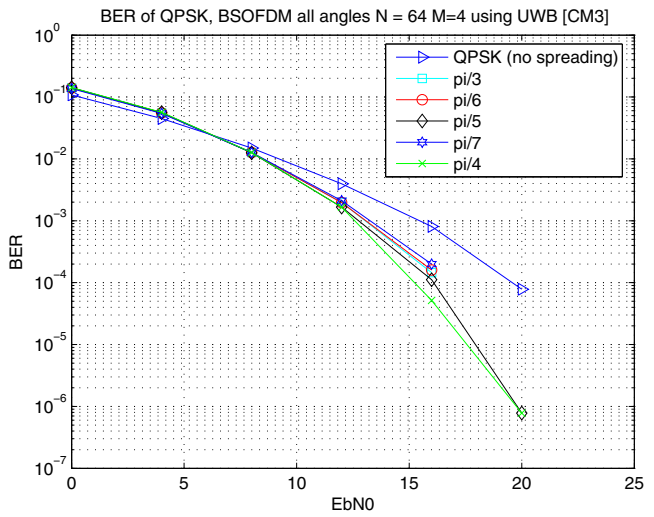

Figure 13. Comparing all angles in UWB $C M 3$, it can be seen that $\frac{\pi}{4}$ has better results for $M=4 N=64$.

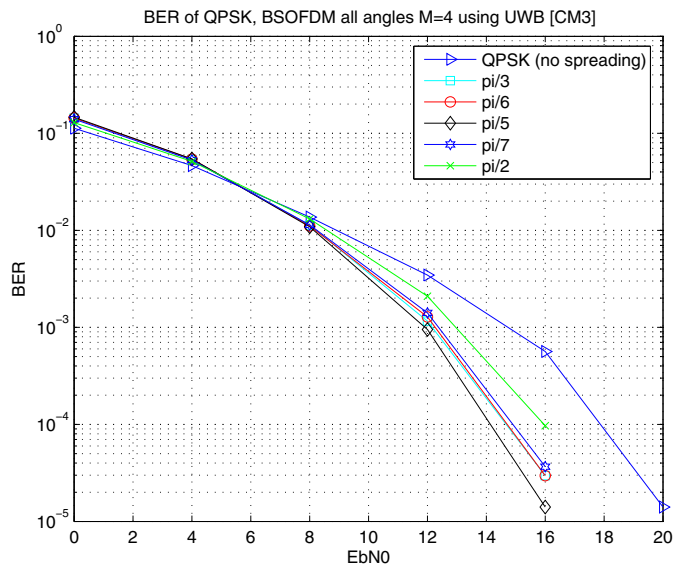

Figure 14. Comparing all angles in UWB $C M 3$, it can be seen that $\frac{\pi}{2}$ although for $M=4$ increases correlation between symbols does not achieve better results.

limits.

\section{Conclusion}

In conclusion this paper presented a study of varying angles for higher order Rotation spreading matrix proposed in [1] and the expansion given in [8] for BSOFDM system over the four UWB channel models proposed for the IEEE.15.3a task group and presented in Table 1. Although in [7] it was shown that for block size of $M=2$ the angles $\alpha=\frac{\pi}{3}$ produced the best result in terms of BER over UWB channels, that was no longer the case for higher order Rotation spreading matrix. It was also shown that although some angles such as $\alpha=\frac{\pi}{4}, \frac{\pi}{2}$ and $\pi$ for $M=2$ were useless due to them simply rotating the modulation scheme back onto it self and did not increase the correlation between the transmitted signals, these same angles for higher order Rotation matrix were shown to not only in-

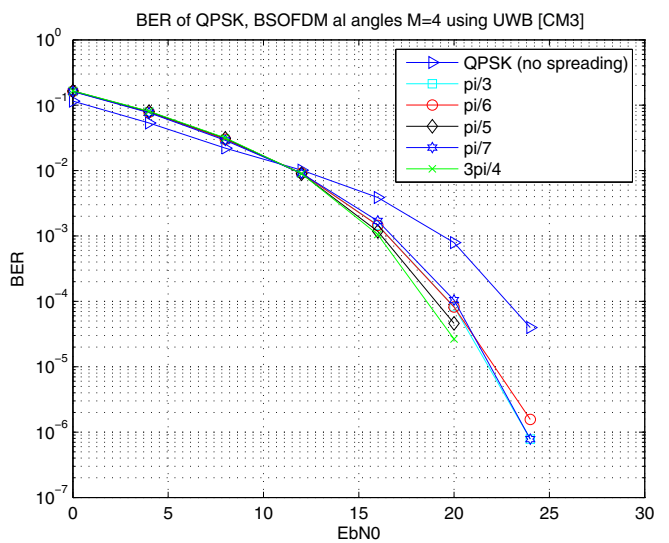

Figure 15. Comparing all angles in UWB $C M 3$, it can be seen that $\frac{3 \pi}{4}$ has better results for $M=4 N=64$.

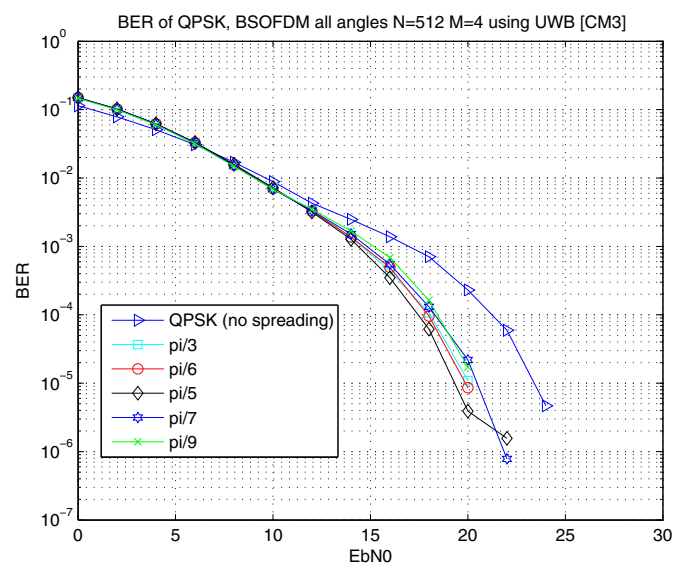

Figure 16. Comparing all angles in UWB $C M 3$, it can be seen that $\frac{\pi}{5}$ has better results, $M=4 N=64$.

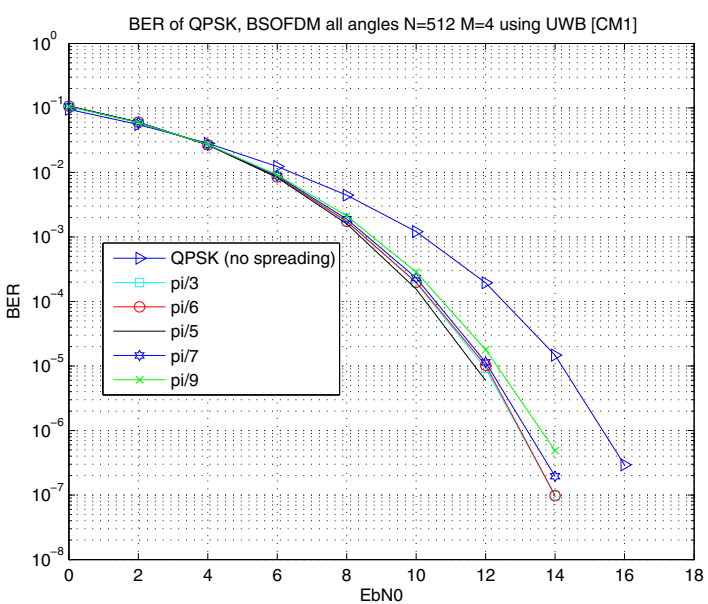

Figure 17. Comparing all angles in UWB $C M 1$, it can be seen that $\frac{\pi}{5}$ has better results for $M=4 N=512$. 
crease the correlation but in some cases such as the angle $\alpha=\frac{\pi}{4}$ outperformed other angles in UWB channels.

It can be said that the previous limitation of the angles mentioned above no longer hold for larger block sizes of $M$ for higher order Rotation spreading matrix. In other words when using for example a QPSK modulation scheme for block size $M=2$, the use of the angle $\alpha=\frac{\pi}{2}$, would simply rotate the modulation on it self and end up using the same modulation scheme again, in higher order this same angle increased the correlation between the transmitted symbols.

Again as in the study presented in [7] it can be stated that the advantages can be noted of the Rotation spreading matrix is its flexibility in determining different structures of matrices and the simple angle rotation allows an improvement to take place over more traditional spreading matrices.

It can be stated at the end of this simple study, that the angles which perform the best over UWB channel models for block size $M=4$ are the angles $\frac{\pi}{5}, \frac{\pi}{6}, \frac{3 \pi}{4}$ and $\frac{\pi}{4}$.

Future work on this Rotation spreading matrix will include finding the theoretical optimum angle to achieve the best result for the higher order Rotation spreading matrix.

\section{References}

[1] Ibrahim Raad, Xiaojing Huang and Raad Raad, "A New Spreading Matrix for Block Spread OFDM" 10th IEEE International Conference on Communication Systems 2006 (IEEE ICCS'06), Singapore, 31 October - 3 November 2006.

[2] Michael L. McCloud, "Coded block OFDM for the frequency selective fading channel”, Signals, Systems and Computers, Conference Record of the Thirty-Eighth Asilomar Conference on, volume 1, 2004, 329-333, November.

[3] Michael L. McCloud, "Analysis and Design of Short Block OFDM Spreading Matrices for Use on Multipath Fading Channels", Communications, IEEE Transactions on, volume 53, 4, 2005, 656-66, April.

[4] Michael L. McCloud, "Optimal Binary Spreading for Block OFDM on Multipath Fading Channels", WCNC / IEEE Communications Society, volume 2, 2004, 965-970, March.

[5] Ibrahim S. Raad, Xiaojing Huang, "Exploiting Time diversity to improve Block Spread OFDM", First IEEE International Conference on Wireless Broadband and Ultra Wideband Communications, Aus Wireless 2006, Sydney, 13 - 16 March.

[6] Ibrahim S. Raad and Xiaojing Huang , "Exploiting Time Diversity to Improve Block Spread OFDM in a Multipath Environment" The Second International Conference on Information \& Communication Technologies - From Theory to Applications, IEEE ICTTA’06, Damascus, Syria, 24 - 28 April 2006.
[7] Ibrahim Raad, Xiaojing Huang and Darryn Lowe, "A Study of different angles for the New Spread Matrix for BSOFDM in UWB channels", accepted for publication in The Third International Conference on Wireless and Mobile Communications ICWMC 2007, March 4-9, 2007 - Guadeloupe, French Caribbean.

[8] Ibrahim Raad, Xiaojing Huang and Darryn Lowe, "Higher Order New Matrix for Block Spread OFDM" 14th International conference on telecommunications (ICT), 8th International conference on Communications (MICC), 14th -17th May 2007, Penang Malaysia.

[9] Darryn W. Lowe , "Real - time FPGA Realization of an UWB transceiver physical layer" Thesis for Masters of Engineering Research at the University of Wollongong, The School of Electrical, Computer and Telecommunications Engineering, 2005. 